\title{
Clinical Usefulness of the Electroencephalogram in Acute Stroke - A Preliminary Study
}

\author{
Mariana Silva $^{1}$, Axel Ferreira ${ }^{2}$, Paulo Coelho ${ }^{2}$, Daniel Filipe Borges ${ }^{1,2,3 *}$, Telmo Pereira ${ }^{1}$ and Jorge Conde ${ }^{1}$ \\ ${ }^{1}$ Department of Clinical Physiology, Polytechnic Institute of Coimbra, Portugal \\ ${ }^{2}$ Department of Neurology and Neurophysiology, Hospital Pedro Hispano - Local Health Unit of Matosinhos, Portugal
}

${ }^{3}$ PhD Program in Neuroscience, Faculty of Medicine - University of Porto, Portugal

*Corresponding author: Daniel Filipe Borges, Department of Clinical Physiology,

Polytechnic Institute of Coimbra and Department of Neurology and Neurophysiology,

Hospital Pedro Hispano, Portugal.

Published Date: October 17, 2018

\begin{abstract}
Introduction: Stroke is the main cause of disability worldwide, being the first cause of death in Portugal. In the first hours of the event, the cranioencephalic CT scan (CT Scan) does not show the lesion in about $74 \%$ of cases, making validation of alternative diagnostic approaches of utmost importance. The electroencephalogram (EEG) may provide useful information for the diagnosis and prognosis of stroke.
\end{abstract}

Objective: To study the potential usefulness of the EEG for the early diagnosis of acute stroke in patients with initial negative CT Scan, and for the evaluation of the functional status and risk of epilepsy.

Methods: Retrospective analysis of patients with ischemic stroke who underwent EEG and acute phase CT scan between January 2014 and February 2018. Patient characteristics and stroke were classified according to the Oxfordshire Community Stroke Project (OCSP) criteria. The patients were functionally evaluated at 12 months post-stroke by the modified Rankin Scale (mRS) and the existence of post-stroke epilepsy was determined by telephone interview on February 2018.

Results: Thirty patients (25 females and 5 males, mean age 70.5 years) were included. According to the OCSP were identified: $40 \%$ TACS, 37\% PACS, 10\% LACS and 13\% POCS. 50\% with acute vascular injury visible on the initial CT Scan performed with 7 hours of evolution in median. All patients underwent EEG with a median of 3 days of evolution, and slow focal activity was observed in all patients, and focal paroxysmal activity (PA) was seen in $17 \%$ of the participants. 17 patients (58\% of patients) developed post-stroke epilepsy (PSE) with 4 of these having PA evidence in the initial EEG (24\%). One of the patients with PA in the initial EEG did not develop epilepsy during a 4 years follow-up period. In patients without PA, the average of mRs at follow-up was 3.8 and the mortality was $24 \%$, whereas in patients with PA, the mean of the mRs was 5.0 and the mortality was $40 \%$.

Conclusion: In this study, unlike CT Scan, the acute-phase EEG presented with abnormal features in all patients with acute stroke, therefore the EEG may potentially provide significant diagnostic information, estimates of risk for developing future epilepsy and also overall risk stratification. Further studies are needed to validate this hypothesis.

Key words: Eletroencephalogram; Stroke; Epilepsy; CT scan

\section{Introduction}

Stroke is defined as the installation of neurological deficit attributable to a focal central nervous system (CNS) lesion of vascular etiology including ischemic phenomena, intracerebral hemorrhage and subarachnoid haemorrhage [1,2]. This clinical entity accounts for about $10 \%$ of all deaths worldwide, the highest in developing countries and the second in countries with high incomes per capita, being also the main direct cause of long-term

disability in adults [3]. About $80 \%$ of patients survive the acute phase of a stroke and 6 months later approximately 85\% still present motor impairment as well as neurological, communication, cognitive and visuospatial deficits [4]. Cerebrovascular diseases have long been the leading cause of death in Portugal. According to the Health General Directorate, during 2014, about 20,000 Portuguese suffered a stroke and the number of deaths due to 
cerebrovascular diseases in 2013 was 11,751 [5]. Stroke is closely related to several well-known cardiovascular risk factors, such as hypertension, diabetes, hyperlipidemia and atrial fibrillation [6]. In adults, the most common causal categories of ischemic stroke are atherosclerosis of large arteries (such as the middle cerebral artery), cardiac embolism, small vessel disease and other causes, including inflammatory disease, hypercoagulable states and arterial dissection [7].

Cerebrovascular disease has a huge social and individual cost. Treatment costs and other associated costs, reported in different studies, are variable as well as the methodologies used in its calculation. Still, as an example, one study [8] estimated costs in the UK at 8.9 billion euros annually, including loss of productivity, from which only $5 \%$ were related to initial treatment. In the United States, annual costs are around $\$ 33$ billion (including medical treatment, medication and productivity breakdown). Therefore, employing reliable and objective techniques for diagnosis and prognosis are necessary in order to enable effective clinical decision-making. The electroencephalogram (EEG) is a low-cost, non-invasive and reproducible tool that allows the acquisition and analysis of brain electrical activity by interpreting the patterns found regarding its morphology, frequency, voltage and topography [9]. This method provides real-time information on the evolution pattern of cortical activation, namely those that can be used as diagnostic and prognostic markers in order to characterize the physiological deficit in earlier stages, and to potentiate the rehabilitation of each patient affected by stroke. The EEG is a method whose sensitivity is already documented in the functional evaluation after ischemic stroke. There is a crucial ischemic limit in which neurons begin to lose their transmembrane gradients leading to cell death (stroke) [10]. This ischemic limit is visible in the EEG due to the physiological coupling of the eletrophysiological activity with cerebral blood flow (CBF) [11]. In the area called the ischemic nucleus, where the blood flow is less than $10 \mathrm{ml} / 100 \mathrm{gr}$ of tissue/min, there is a dysfunction of cellular metabolism leading to an influx of calcium ions and to an accumulation of water in the intracellular space. This phenomenon is called cytotoxic edema and occurs early in cerebral ischemia. Ischemia affects the blood-brain barrier allowing blood proteins and water to enter the extracellular space leading to vasogenic edema. Thus, recent studies have shown that cerebral ischemia causes a permanent or transient functional rupture in cerebral connections, especially in the gray matter where the cells of the neuroglia are located, both at the local level of the lesion and at the distal level [2]. When the CBF decreases to approximately $25-35 \mathrm{ml} / 100 \mathrm{~g} / \mathrm{min}$, the EEG initially loses faster frequencies, with the slower frequencies increasing gradually when CBF decreases to approximately $17-18 \mathrm{ml} / 100 \mathrm{~g} / \mathrm{min}$. The stroke may not occur for hours with this degree of flow limitation and some electrical activity can be observed (mainly delta frequencies), but as the CBF continues to decline towards the infarction threshold (10-12 $\mathrm{ml} / 100 \mathrm{~g} / \mathrm{min}$ and below), the EEG becomes hypovolemic and cell lesions become irreversible [10,12,13].

EEG is often used to diagnose epilepsy secondary to a stroke since it is essential in detecting ictal and inter ictal activity in epileptiform activity. This tool allows to study the characteristics and clinical results of the patients, monitoring the effect of the different antiepileptic drugs [14]. In fact, stroke is a common epileptogenic cause and up to $30 \%$ of patients who have suffered a stroke have developed post-stroke epilepsy (PSE) [15-19]. The majority of cases of PSE are due to arterial ischemic stroke [20]. A strategy for recognizing and preventing acute symptomatic seizures may be clinically relevant since these may be independent predictors of functional outcome in the first year after stroke [21]. Thus, the early presentation of a seizure refers to an event of epileptic etiology that occurs during the first week after stroke acute symptomatic seizure. The late epileptic seizure occurs more than one week after stroke. Most patients with early seizures do not develop late epileptic seizures or recurrent epilepsy. The also allows studying the characteristics of individual patients by monitoring the effect of the different antiepileptic drugs [14]. Thus, it enables the investigation of brain areas with epileptogenesis potential after ischemic stroke, allowing functionally assessment in different stages of the process $[22,23]$.

The aim of our study was to document the existence of abnormal changes in the EEG patterns in acute stroke, namely with regards to its electrophysiological and topographic characteristics during the injury process. The research hypothesis is based on the potential role of the EEG as a diagnostic and risk stratifying tool, when used early on suspicious acute ischemic stroke.

\section{Methods}

We performed a retrospective analysis of clinical data from patients who underwent EEG and cranioencephalic computed tomography (CT Scan) in the acute phase of suspected ischemic stroke, in the Neurology Department of Local Health Unit of Matosinhos - Hospital Pedro Hispano between January 2014 and February 2018.

The patients were classified according to the criteria of the Oxfordshire Community Stroke Project (OCSP) [24], which allows for a syndromic characterization of the stroke (LACS-Lacunar Syndrome, TACS-Total Anterior Circulation Stroke, PACS-Partial Anterior Circulation Syndrome, POCS-Posterior Circulation Syndrome). Functional prognosis of patients was measured through the modified Rankin Scale (mRs) [25] at 12 months, as well as the existence of post-stroke epilepsy.

Cardiovascular risk factors and previous clinical events were collected including the presence of hypertension, diabetes mellitus, dyslipidemia, smoking habits, use of oral contraceptives, personal and family history of cerebrovascular or cardiac disease. The presence of hypertension, diabetes mellitus and dyslipidemia were based on each patient on-going medication and clinical record.

All EEG recordings complied with the Department protocol and the International recommendations. From the EEG, the existence and topography of slow focal and/or paroxysmal activity was verified and correlated with the individual clinical profile and CT Scan lesions.

Data collection was performed at the Local Health Unit of Matosinhos - Hospital Pedro Hispano. The process will consist of obtaining and placing the above parameters in a single database. 
All variables were entered in a Microsoft Excel 2016 database using Microsoft Excel 2016 software. Variables were organized, codified and anonymized. The study was approved by the Ethics Committee and Board of Directors of the Local Health Unit of Matosinhos.

\section{Statistical analysis}

Data were computerized and treated with SPSS (Statistical Package for Social Sciences), version 24.0 (IBM, Armonk, NY). The distribution of variables was tested for normality using the ShapiroWilks test, and homogeneity of variance by Levene's test. Simple Descriptive statistics were used to characterize the sample and the distribution of variables. Data are presented as mean \pm standard deviation (SD) for continuous variables and as a frequency (\%) for categorical variables. Continuous variables were compared using the Student t-test, and categorical variables were compared using the Fisher's exact test. A p-value $\leq 0.05$ was set as the criterion for statistical significance, for a confidence interval (CI) 95.

\section{Results}

\section{Demographic data}

A total of 30 patients (25 women - 83.3\%) diagnosed with ischemic stroke were included in this study. The ages of the patients ranged from 18 to 92 years, with an average age of 70.5 (SD = 19.2) years. $63.3 \%$ of the patients were over 65 years old. Cardiovascular risk factors were present in the majority of the study population (93.3\%), and clustering of risk factors were identified in 10 patients (33.3\%). The most common cardiovascular risk factor for all individuals was arterial hypertension (HT), observed in $76.7 \%$ of the patients. Dyslipidemia was observed in $46.7 \%$ of patients, 20\% had Type II Diabetes Mellitus, 13.3\% were obese, 10\% were current smokers and $23.3 \%$ had atrial fibrillation (Table 1).

Table 1:

\begin{tabular}{|c|c|}
\hline Cardiovascular Risk Factors & N (\%) \\
\hline Arterial hypertension & $23(76,7 \%)$ \\
\hline Dyslipidemia & $14(46,7 \%)$ \\
\hline Atrial Fibrillation & $7(23,3 \%)$ \\
\hline Diabetes Mellitus & $6(20 \%)$ \\
\hline Obesity & $4(13,3 \%)$ \\
\hline Smoking & $3(10 \%)$ \\
\hline Alcoholism & $1(3,33 \%)$ \\
\hline Oral Contraceptive & $1(3,33 \%)$ \\
\hline Ex-smoker & $1(3,33 \%)$ \\
\hline Heart Failure & $1(3,33 \%)$ \\
\hline
\end{tabular}

\section{Diagnostic data}

\section{Age classes and gender distribution of patients with ischaemic stroke}

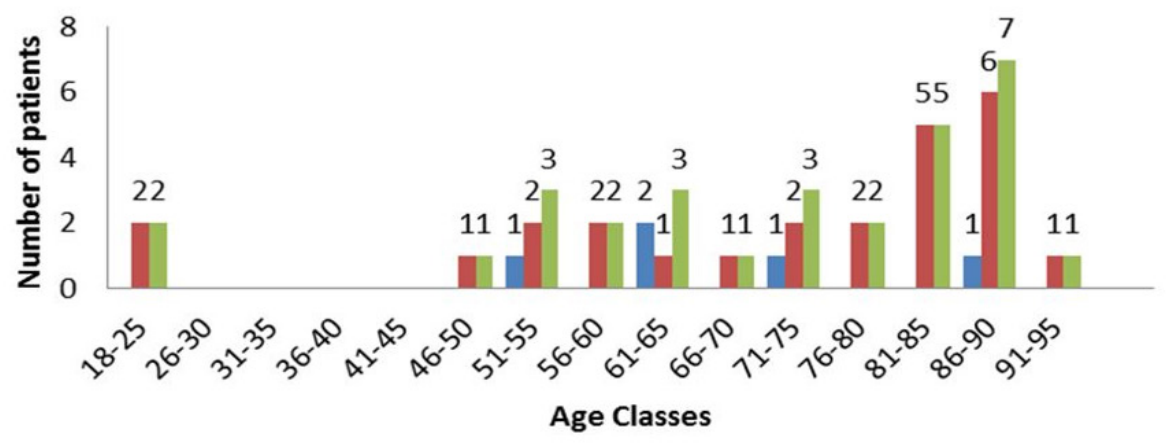

Male Female $=$ Total

Figure 1: Age classes and gender distribution of patients with ischaemic stroke.

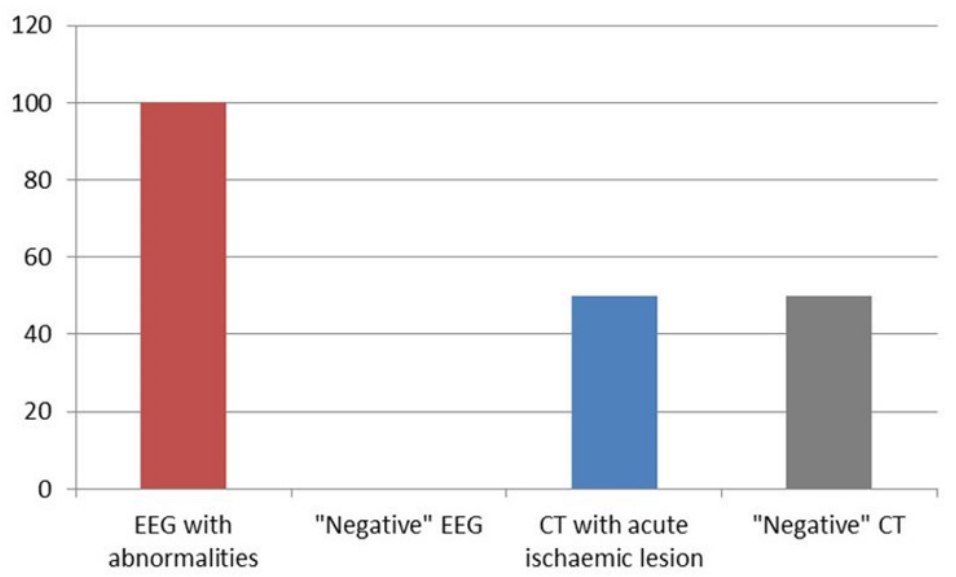

Legend: EEG - electroencephalogram; CT - cranioencephalic computed tomography

Figure 2: Initial results from the EEG and CT scan. 
All patients underwent CT scan and EEG in the acute phase. 15 patients (50\%) presented acute vascular lesion visible at the initial CT Scan, performed in average at 16 hours of evolution from the ictus (range: 0.5-81 hours). All patients underwent a second postevent EEG, on average up to 4 days after the ictus (range: 1-14 days), with electrophysiological abnormalities found in all patients (Figure 1). A second CT Scan was performed in Figure 2 patients who initially had no acute ischemic injury. Of the 15 patients who performed the second imaging examination, the presence of acute vascular injury was confirmed in all patients (Figure 1\&2).

The abnormalities identified in the EEG, depicted in Figure 2, were focal slow activity (SA), in 25 patients (83\%), and slow focal activity and paroxysmal activity (PA), in the remaining 5 patients (17\%) (Figure 3).

\section{Paroxysmal Activity (PA) + Focal Slow Activity (SA) = Focal Slow Activity (SA)}

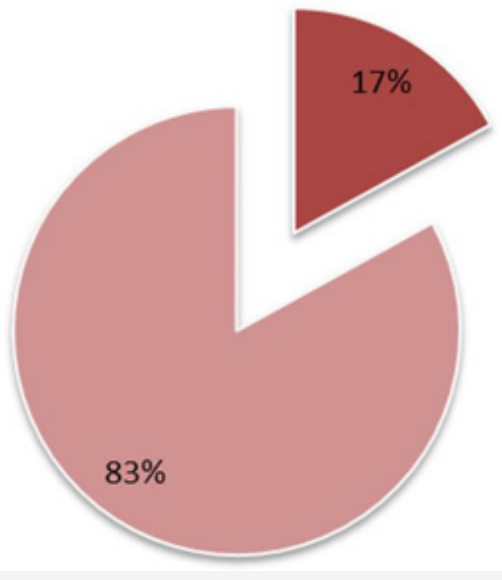

Figure 3: Abnormalities documented by the EEG.

\section{Stroke classification}

According to their location and the criteria of the Oxfordshire Community Stroke Project, $77 \%$ of the ischemic strokes were dependent on the impairment of the anterior circulation, corresponding to TACS in $40 \%$ of the patients and PACS in $37 \%$. POCS and LACS were identified in $13 \%$ and $10 \%$ of the patients, respectively (Figure 4).

Legend: TACS: Total anterior circulation stroke; PACS: Partial anterior circulation syndrome; LACS: Lacunar Syndrome; POCS: Posterior Circulation Syndrome

Figure 4: Stroke Classification according to the Oxfordshire Community Stroke Project criteria.

\section{Post-Stroke functional evaluation-modified rankin scale}

In all patients, functional status was assessed according to the Modified Rankin Scale. In the presence of paroxysmal activity on the EEG, patients were classified in three grades, with the highest severity points of the scale (4 - moderately severe deficiency, 5 severe deficiency, 6 - death). The patients without paroxysmal activity on the EEG, were classified with the score of mRS ranging from 0 to 6 . The mortality rate was $30 \%$ and $26.7 \%$ of patients had Rankin 1-3 (Figure 5). The average initial mRS was 1.3 (SD: 1.6; range: $0-5.0$ ), increasing significantly to an average mRS of 4.1 (SD: 1.9; range: $0-6.0)$ after 1 -year follow-up $(p<0.001)$, with similar trend in males and females (Figure 5). 


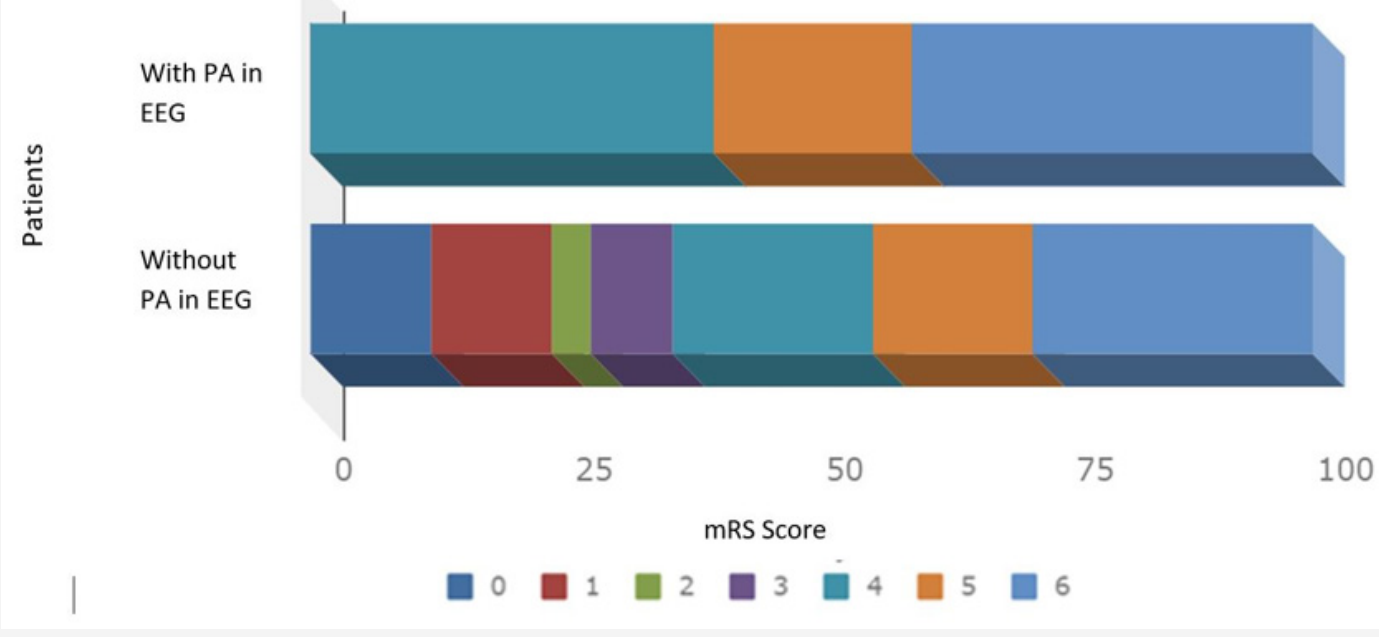

Figure 5: Functional evaluation, at 12 months, by Modified Rankin Scale (mRS).

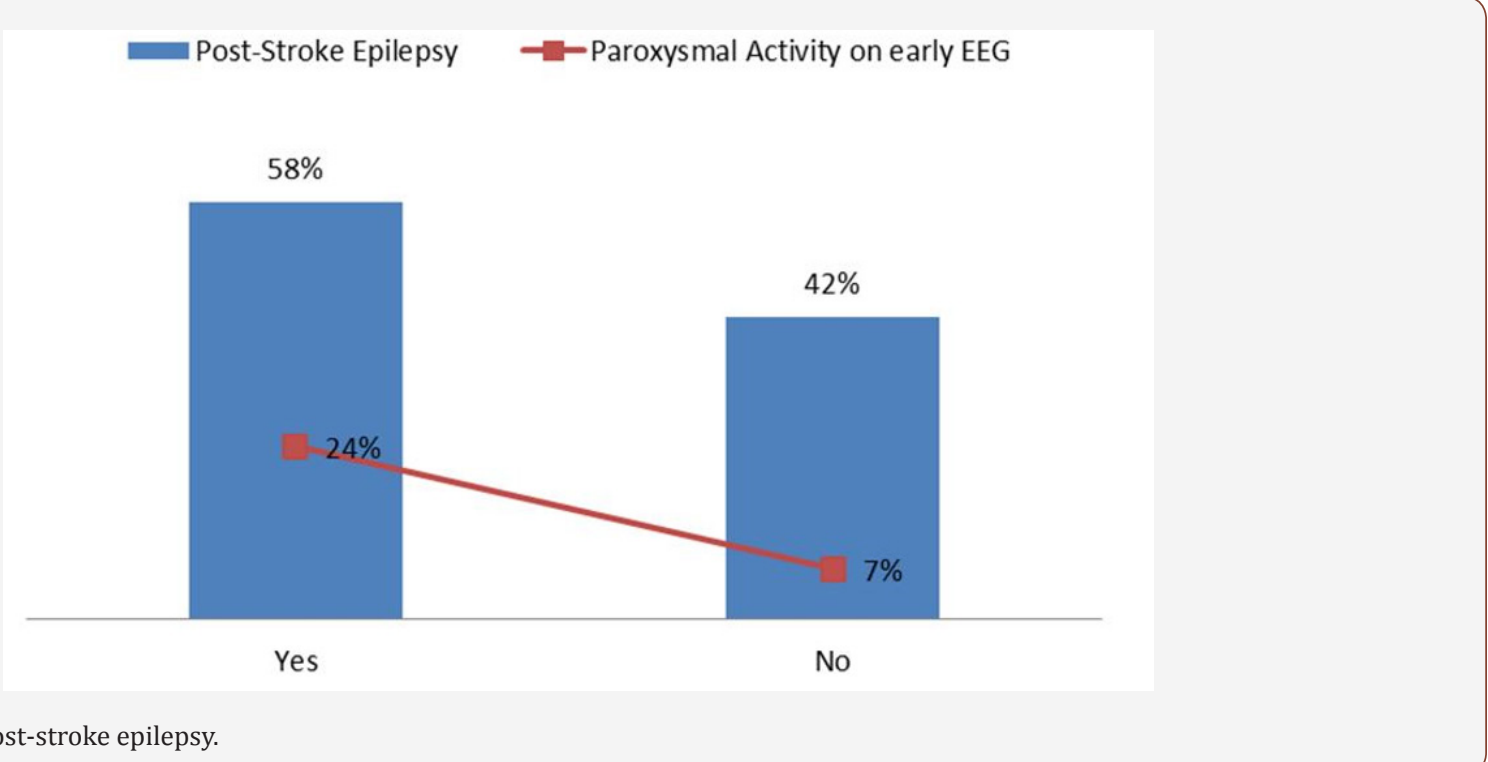

Figure 6: Presence of post-stroke epilepsy.

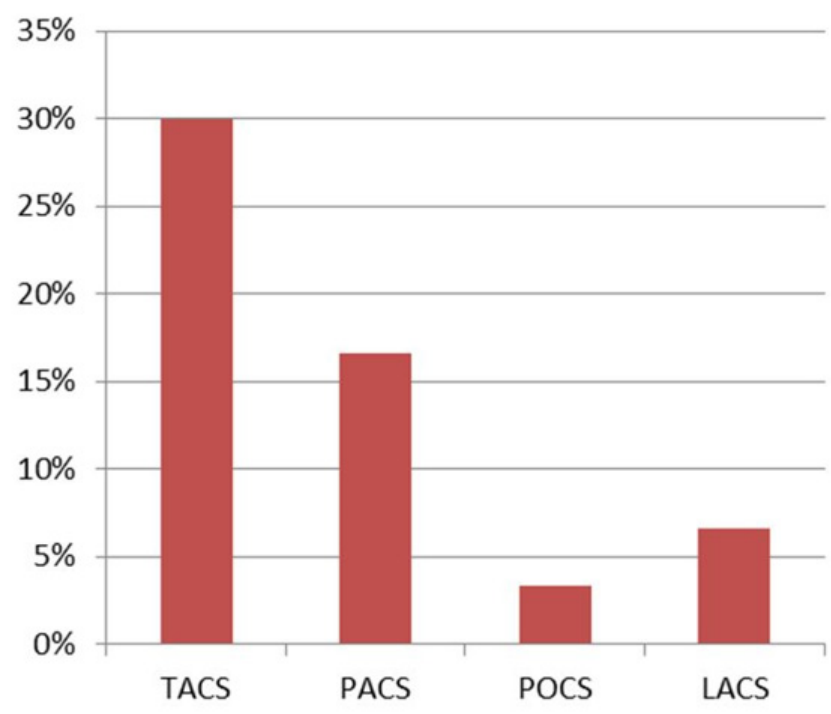

Figure 7: Post-stroke epilepsy according to stroke classification (by OCSP). 
Regarding post-stroke epilepsy, its existence in follow-up was crossed with the evidence of PA in the initial EEG. Overall, vascular epilepsy was observed in $58 \%$ of the patients, and approximately half of them presented with PA in the initial EEG (Figure 6). Conversely, only $7 \%$ of the patients without post-stroke epilepsy presented PA in the initial EEG. Considering the occurrence of vascular epilepsy as a function of the Oxfordshire Community Stroke Project criteria, a greater association was found with the anterior circulation compromise, representing a total of over $80 \%$ of the patients with either TACS or PACS and vascular epilepsy during the follow-up $(\mathrm{p}<0.05)$ (Figure 7).

\section{Discussion}

The role of the EEG in the early evaluation of stroke is yet to be fully understood, although some evidences provide support to its application. Considering the results observed in this preliminary study, one striking finding was the presence of slow focal activity in all patients, even in those with normal initial CT scan. Other studies have also reported the presence of this electrophysiological pattern restricted to the hemisphere that suffers from ischemia [26,27]. As described in the literature, the CT scan is often a limited tool in the diagnosis of acute ischemic stroke, failing to show lesions in the first 6 hours in up to 74\% of the stroke cases [28], and therefore, the validation of alternative diagnostic procedures would be of utmost importance for clinical decision.

In our study, the acute-phase EEG presented with abnormalities in all patients, unlike CT scan, raising the question to whether a diagnostic role for this method could be expected. Other small studies have addressed this issue with quite promising results. A study using quantitative EEG indexes in stroke patients demonstrated that, when used in an appropriate time frame, the EEG have a better performance than a clinical scale in predicting the end result [29], while other study demonstrated a better correlation of the acute phase EEG with neuroimaging lesions after stroke compared with acute phase magnetic resonance imaging [30]. In the clinical practice of most stroke units, only a second imaging test (CT scan) is performed on patients who undergo intravenous treatment or who present worsening of neurological symptoms [21].

In our study population, the 15 patients who underwent an acute phase image examination without presenting acute vascular injury performed a second imaging test that confirmed the presence of acute ischemic injury which, in a way, validates and correlates topographically with the EEG findings. In addition, the EEG detected PA in about $1 / 5$ of the patients, a risk factor for developing epilepsy and for worst prognosis during follow-up. In fact, the EEG is the method of choice to diagnose epilepsy secondary to stroke.

According to Bentes et al. [21], a strategy for recognizing and preventing acute symptomatic seizures may be clinically relevant, since acute symptomatic seizures and seizures may be independent predictors of functional outcome in the first year after stroke, and consequently, of quality of life [21].

Stroke is a common epileptogenic cause, accounting for up to $9 \%$ incident cases of epilepsy [31]. In our study population, $58 \%$ of the patients developed vascular epilepsy. Of these patients, $24 \%$ had
PA in the acute phase EEG. The clinical severity of stroke, regardless of the scale used, is also an important factor in the development of PSE [15,19,32-34]. Total anterior circulation stroke is a particularly important risk factor for post-stroke epileptogenesis compared to other ischemic stroke subtypes [35-38]. In our study, $40 \%$ of patients suffered a TACS, and the results were in agreement with what was described in the literature. Most of the patients developing epilepsy during the follow-up had anterior circulation impairment.

Going to the pivotal notion that "Time is Brain", we must bear in mind that an estimated neuronal loss of 1.2 billion and a brain aging of 36 years is expected in stroke [39]. Thus, achieving early diagnosis of the event is of paramount importance, considering that it will allow for early treatment of the vascular condition. From our results, it appears that some features of the EEG are closely related to the clinical severity and topography of the stroke, and may be a sensitive neurological diagnostic technique, not only to determine the functional outcome of the event [21], but also to provide diagnostic cues to the identification a stroke, even, and specially, when the CT scan fails to provide a positive result, which was seen in a significant proportion of the included patients and could account for up to $74 \%$ of the cases in the first 6 hours of an acute ischemic stroke[28]. In our study population, the 15 patients who underwent an acute phase image examination without presenting acute vascular injury performed a second imaging test that confirmed the presence of acute ischemic injury which, in a way, validates and correlates topographically with the EEG findings. Further studies are needed to identify the EEG traits that could be potential markers of stroke, and thus, convey diagnostic value to clinical decision [40-43].

\section{Conclusion}

The retrospective nature of the study is a major limitation, as well as the small subset of participants included. Notwithstanding, our research provides relevant preliminary information regarding the potential diagnostic capabilities of the EEG for the early phases of stroke. The early neurophysiological approach in this subtype of patients, through the use of EEG in the acute phase of vascular encephalic injury, has the potential to be informative, particularly when neuroimaging data are negative. Consecutive use of the EEG in the context of stroke additionally provides the unusual possibility, at a low cost, of deepening the neurophysiological knowledge of cortical dynamics, which in fact can be extraordinarily fruitful for risk stratification and prognosis inherent to stroke.

In summary, our preliminary data provide evidence in favor of the use of the EEG as an evaluation tool in the early phases of stroke. Further studies are warranted to identify electrophysiological abnormalities or adaptations that could provide added diagnostic or prognostic information, thus contributing to better clinical decision-making.

\section{Acknowledgement}

None.

\section{Conflict of Interest}

No conflict of interest. 


\section{References}

1. Aho K et al. (1980) Bull World Health Organ 58: 113-130.

2. Palm F, Urbanek C, Wolf J, Buggle F, Kleemann T, et al. (2012) Etiology, risk factors and sex differences in ischemic stroke in the Ludwigshafen Stroke Study, a population-based stroke registry. Cerebrovasc Dis 33(1): 69-75.

3. Carod-Artal J, Egido JA, González JL, Varela de Seijas E (2000) Quality of life among stroke survivors evaluated 1 year after stroke: experience of a stroke unit. Stroke 31(12):2995-3000.

4. Duncan PW, Zorowitz R, Bates B, Choi JY, Glasberg JJ, et al. (2005) Management of adult stroke rehabilitation care: a clinical practice guideline. Stroke 36(9): 100-143.

5. Direcção Geral da Saúde (DGS) (2015) Portugal. Doenças CérebroCardiovasculares em Números.

6. Park TH, Ko Y, Lee SJ, Lee KB, Lee J, et al. (2015) Identifying target risk factors using populationattributable risks of ischemicstrokebyageandsex. J Stroke 17: 302-311.

7. Adams HP, Biller J (2015) Classification of subtypes of ischemic stroke: history of the trial of org 10172 in acute stroke treatment classification. Stroke 46(5): 114-118.

8. Feigin VL, Forouzanfar MH, Krishnamurthi R, Mensah GA, Connor $M$, et al. (2014) Global and region al burden of stroke during 19902010: findings from the Global Burden of Disease Study 2010. Lancet 383(9913): 245-254

9. Monge-Pereira E, Molina-Rueda F, Rivas-Montero FM, Ibáñez J, Serrano JI, et al. (2017) Electroencefalografía como método de evaluación tras un ictus. Una revisión actualizada. Neurología 32(1): 40-49.

10. Strong K, Mathers C, Bonita R (2007) Preventing stroke: saving lives around the world. Lancet Neurol 6(2): 182-187.

11. Faught E (1993) Current role of elecroencephalography in cerebral ischemia. Stroke 24(4): 609-613.

12. Sharbrough FW, Messick JM Jr, Sundt TM Jr (1973) Correlation of continuous electroencephalograms with cerebral blood flow measurements during carotid endarterectomy. Stroke 4: 674-683.

13. Gawryluk JR, D’Arcy RC, Connolly JF, Weaver DF (2010) Improvingthe clinical assessment of consciousness with advances in electrophysiological and neuroimaging techniques. BMC Neurol 29: 10-11.

14. Jordan KG (2004) Emergency EEG and continuous EEG monitoring in acute ischemic stroke. J Clin Neurophysiol 21(5): 341-352.

15. Hossmann KA (1994) Viability thresholds and the penumbra of focal ischemia. Ann Neurol 36(4): 557-565.

16. Temprano T, Salas-Puig J, Calleja-Puerta S, Zanabili Al-SibbaiAA, Lahoz CH (2009) Epilepsia postictus. Rev Neurol 48:171-177.

17. Bladin CF, Alexandrov AV, Bellavance A, Bornstein N, Chambers B, et al. (2000) Seizures after stroke: a prospective multicenter study. Arch Neurol 57(11): 1617-1622.

18. Menon B, Shorvon SD (2009) Ischaemic stroke in adults and epilepsy. Epilepsy Res 87(1): 1-11

19. Jungehulsing GJ, Heuschmann PU, Holtkamp M, Schwab S, KolominskyRabas PL (2013) Incidence and predictors of post-stroke epilepsy. Acta Neurol Scand 127(6): 427-430.

20. Graham NSN, Crichton S, Koutroumanidis M, Wolfe CD, Rudd AG (2013) Incidence and associations of poststroke epilepsy the prospective South London stroke register. Stroke 44(3): 605-611.

21. Bentes C, Peralta AR, Martins H, Casimiro C, Morgado C, et al. (2017) Seizures, electroencephalographic abnormalities, and outcome of ischemic stroke patients. Epilepsia Open 2(4): 441-452.

22. Teasdale G, Jennett B (1974) Assessment of coma and impaired consciousness. A practical scale. Lancet 2(7872): 81-84.
23. Beghi E, Carpio A, Forsgren L, Hesdorffer DC, Malmgren K, et al. (2010) Recommendation for a definition of acute symptomatic seizure. Epilepsia 51(4): 671-675.

24. Bamford J, Sandercock P, Dennis M, Burn J, Warlow C (1991) Classification and natural history; of clinically identifiable subtypes of cerebral infarction. Lancet 337(8756): 1521-1526.

25. Bonita R, Beaglehole R (1988) Modification of Rankin Scale: Recovery of motor function after stroke. Stroke 19(12): 1497-1500.

26. Cardoso T, Fonseca T, Costa M (2003) Acidente Vascular Cerebral no Adulto Jovem. Acta Médica Portuguesa 16: 239-244.

27. De Reuck J, Goethals M, Claeys I, Van Maele G, De Clerck M (2006) EEG findings after a cerebral territorial infarct in patients who develop earlyand late-onset seizures. Eur Neurol 55: 209-213.

28. Graham NS, Crichton S, Koutroumanidis M, Wolfe CD, Rudd AG (2013) Incidence and associations of poststroke epilepsy the prospective South London stroke register. Stroke 44(3): 605-611.

29. Chalela JA, Kidwell CS, Nentwich LM, Luby M, Butman JA, et al. (2007) Magnetic resonance imaging and computed tomography in emergency assessment of patients with suspected acute stroke: a prospective comparison. Lancet 369(9558): 293-298.

30. Cuspineda E, Machado C, Aubert E, Galán L, Llopis F, et al. (2003) Predicting outcome in acute stroke: a comparison between QEEG and the Canadian Neurological Scale. Clin Electroencephalogr 34: 1-4.

31. Finnigan SP, Rose SE, Walsh M, Griffin M, Janke AL, et al. (2004) Correlation of quantitative EEGin acute ischemic stroke with 30-day NIHSS score: comparison withdiffusion and perfusion MRI. Stroke 35(4): 899-903.

32. Adelow C, Andersson T, Ahlbom A, Tomson T (2011) Prior hospitalization for stroke, diabetes, myocardial infarction, and subsequent risk of unprovoked seizures. Epilepsia 52(2): 301-307.

33. Jungehulsing GJ, Heuschmann PU, Holtkamp M, Schwab S, KolominskyRabas PL (2013) Incidence and predictors of post-stroke epilepsy. Acta Neurol Scand 127(6): 427-430.

34. Arntz R, Rutten-Jacobs L, Maaijwee N, Schoonderwaldt H, Dorresteijn L, et al. (2013) Post-stroke epilepsy in young adults: a long-term follow-up study. PLoS One 8(2): e55498.

35. Burn J, Dennis M, Bamford J, Sandercock P, Wade D, et al. (1997) Epileptic seizures after a fi rst stroke: the Oxfordshire Community Stroke Project. BMJ 315: 1582-1587.

36. De Reuck J, Goethals M, Claeys I, Van Maele G, De Clerck M (2006) EEG findings after a cerebral territorial infarct in patients who develop earlyand late-onset seizures. Eur Neurol 55(4): 209-213.

37. Graham NS, Crichton S, Koutroumanidis M, Wolfe CD, Rudd AG (2013) Incidence and associations of poststroke epilepsy the prospective South London stroke register. Stroke 44(3): 605-611.

38. Roivainen R, Haapaniemi E, Putaala J, Kaste M, Tatlisumak T (2013) Young adult ischaemic stroke related acute symptomatic and late seizures: risk factors. Eur J Neurol 20: 1247-1255.

39. Saver JL (2006) Time is brain-quantified. Stroke 37(1): 263-266.c

40. Beghi E, Carpio A, Forsgren L, Hesdorffer DC, Malmgren K, et al. (2010) Recommendation for a definition of acute symptomatic seizure. Epilepsia 51(4): 671-675.

41. Teasdale G, Jennett B (1974) Assessment of coma and impaired consciousness. A practical scale. Lancet 2(7872): 81-84.

42. Elaborado pela DSIA com base em dados cedidos pelo INE, 2015

43. Bronner LL, Kanter DS, Manson JE (1995) Primary Prevention of Stroke. NEJM 333: 1392-1400. 